\title{
Augmentation of CD47-SIRPa signaling protects cones in genetic models of retinal degeneration
}

Sean K. Wang, ${ }^{1,2}$ Yunlu Xue, ${ }^{1,2}$ Constance L. Cepko ${ }^{1,2,3}$

${ }^{1}$ Department of Genetics, Blavatnik Institute, Harvard Medical School, Boston, MA 02115, USA

${ }^{2}$ Department of Ophthalmology, Harvard Medical School, Boston, MA 02115, USA

${ }^{3}$ Howard Hughes Medical Institute, Chevy Chase, MD 20815, USA

\section{Corresponding author:}

Constance L. Cepko

Harvard Medical School

77 Avenue Louis Pasteur

NRB Room 360

Boston, MA 02115

$617-432-7618$

cepko@genetics.med.harvard.edu

Conflict of interest statement: The authors have declared that no conflict of interest exists.

Keywords: CD47, SIRP $\alpha, \mathrm{AAV}$, gene therapy, microglia, retinitis pigmentosa, retinal degeneration, photoreceptors 


\begin{abstract}
Inherited retinal diseases such as retinitis pigmentosa $(\mathrm{RP})$ can be caused by thousands of different mutations, a small number of which have been successfully treated with gene replacement. However, this approach has yet to scale and may be infeasible in many cases, highlighting the need for interventions that could benefit more patients. Here, we found that microglial phagocytosis is upregulated during cone degeneration in RP, suggesting that expression of “don't eat me" signals such as CD47 might confer protection to cones. To test this, we delivered an adeno-associated viral (AAV) vector expressing CD47 on cones, which promoted cone survival in three mouse models of RP and preserved visual function. Cone rescue with CD47 required signal regulatory protein alpha (SIRP $\alpha$ ) but not microglia or thrombospondin-1 (TSP1), suggesting that CD47 interacts with SIRP $\alpha$ on non-microglial cells to alleviate degeneration. These findings establish augmentation of CD47-SIRP $\alpha$ signaling as a potential treatment strategy for RP and possibly other forms of neurodegeneration.
\end{abstract}




\section{INTRODUCTION}

A major obstacle in developing treatments for inherited retinal diseases (IRDs) is the enormous genetic heterogeneity of pathogenic mutations. In the largest family of these disorders, retinitis pigmentosa $(\mathrm{RP})$, thousands of unique mutations have been identified spanning $~ 100$ different genes (https://sph.uth.edu/retnet/). For a minority of IRDs, gene replacement therapy with adenoassociated viral (AAV) vectors has led to promising results in clinical trials, and in one instance, a successful commercial drug $(1,2)$. Nonetheless, this strategy has yet to reach the vast majority of patients and may not be feasible for those with less common, gain-of-function, or unidentified mutations.

Given these challenges, another approach to treating RP and other IRDs could be to develop mutation-agnostic therapies that preserve cone photoreceptors. In RP, disease mutations, many of which are exclusively expressed in rods, lead to the early death of these cells, often before the condition is diagnosed (3). Rod death is in itself tolerable, as rods are only needed for vision in dim light. However, rod loss is followed by the degeneration of cones, resulting in deterioration of daylight, color, and high-acuity vision. The reasons for secondary cone degeneration in RP remain incompletely understood, but likely include increased oxidative stress $(4,5)$, decreased glucose availability $(6-8)$, and immune dysregulation $(9,10)$. Regardless of the exact etiology, an intervention capable of protecting cones in RP independent of the initial genetic lesion would potentially be beneficial for many patients.

One of the hallmarks of neurodegenerative disorders including RP is the activation of microglia, the resident immune cells of the retina and central nervous system $(11,12)$. This activation 
upregulates phagocytosis and inflammatory cytokine production, facilitating the clearance of infections and cell debris, but can also damage nearby healthy tissue. In mice, activated microglia during the early stages of RP can engulf living rods, thereby hastening rod death (13, 14). While microglia are similarly activated during cone degeneration $(9,10)$, their impact on cone survival appears to be more nuanced. Previously, we showed that the anti-inflammatory cytokine TGF- $\beta 1$ requires microglia to preserve cones in RP mouse models (10), indicating that microglia are capable of conferring protection to cones. However, we also found the net effect of microglia depletion on cone degeneration to be minimal $(9,10)$, suggesting that any beneficial activities of microglia on cones are likely counter-balanced by detrimental ones. These findings left open the role of microglia in cone death and led us to ask whether microglial phagocytosis might contribute to cone demise.

Here, we tested if expression of an anti-phagocytic molecule on cones could slow the rate of cone degeneration. Specifically, we used an AAV vector encoding CD47, a "don’t eat me" signal known to inhibit engulfment via interaction with signal regulatory protein alpha (SIRP $\alpha$ ) on phagocytic cells $(15,16)$. Expression of CD47 preserved cones and vision in multiple mouse models of RP through a mechanism dependent on SIRP $\alpha$, but surprisingly not microglia. Our findings support augmentation of CD47-SIRP $\alpha$ signaling as a possible mutation-agnostic therapy for RP and potentially other neurodegenerative diseases. 


\section{RESULTS}

\section{Microglia show increased phagocytic activity during secondary cone degeneration}

We previously generated $r d 1 ; \mathrm{CX} 3 \mathrm{CR} 1^{\mathrm{GFP} /+}$ and sighted $\mathrm{CX} 3 \mathrm{CR} 1^{\mathrm{GFP} /+}(r d 1$ heterozygous) mice by crossing the widely used $r d 1$ model of $\mathrm{RP}$ with $\mathrm{CX} 3 \mathrm{CR} 1^{\mathrm{GFP}}$ animals, in which microglia express GFP $(9,17,18)$. To assess if microglia during the later stages of RP might phagocytose cones, retinas from these mice were immunostained for cone arrestin, a marker of all cones. In sighted CX3CR $1^{\mathrm{GFP} /+}$ retinas, microglia lacked any appreciable contact with cone arrestinpositive cells (Figure 1A), consistent with the normal exclusion of microglia from the photoreceptor layer (19). In contrast, microglia in $r d 1$;CX3CR $1^{\mathrm{GFP} /+}$ retinas were often directly adjacent to degenerating cones, although in no case was overt engulfment of cones observed. To more sensitively measure microglial phagocytic activity, we explanted retinas from $r d 1 ; \mathrm{CX} 3 \mathrm{CR} 1^{\mathrm{GFP} /+}$ and sighted $\mathrm{CX} 3 \mathrm{CR} 1^{\mathrm{GFP} /+}$ animals and incubated them with yeast particles conjugated to pHrodo Red, a pH-dependent dye that fluoresces upon lysosomal acidification (Figure 1B) (20). Microglia from these retinas were then analyzed by flow cytometry at postnatal day 20 (P20), the approximate age at which cone death in $r d 1$ mice begins (6), as well as P50, after substantial cone loss has occurred. At both time points, microglia from $r d 1$;CX $3 \mathrm{CR} 1^{\mathrm{GFP} /+}$ retinas internalized significantly more yeast than those from sighted controls (Figure 1C), suggesting persistent elevation of microglial uptake during the later stages of RP. Microglia thus exhibit increased phagocytic activity during secondary cone degeneration, a factor we hypothesized might worsen cone demise.

\section{Expression of the CD47 “don't eat me” signal promotes survival of degenerating cones}


Among the key regulators of phagocytosis are "don't eat me" signals such as CD47, which when present on cells, impede their engulfment by macrophages (21). To test if inhibiting phagocytosis during RP might benefit cones, we created an AAV vector (AAV8-RedO-CD47) using the human red opsin promoter to express CD47 on cones (Figure 2A). In wild-type mice injected subretinally with a GFP control vector (AAV8-RedO-GFP), which labels both M- and S-type cones (8), endogenous CD47 could be seen in retinal plexiform layers as previously described, but not in photoreceptors (Figure 2B) (22). Following co-administration of AAV8-RedO-GFP plus AAV8-RedO-CD47, CD47 immunostaining could additionally be detected in cones. Notably, use of AAV8-RedO-CD47 in wild-type mice produced no obvious changes in cone or retinal morphology when evaluated one month later.

In mouse models of RP, cone degeneration proceeds from the optic nerve head outward with relative sparing of the peripheral retina. To measure the effect of CD47 on cone survival, GFPpositive cones in the central retinas of $r d l$ mice were quantified. Cone counting using a GFP vector has been previously validated by our group and provides unambiguous labeling of cones, enabling automated quantification $(4,9,10,23)$. At these doses, co-infection with two serotype 8 vectors in cones is expected to be at least $90 \%$ (4). Compared to AAV8-RedO-GFP alone, coinfection with AAV8-RedO-CD47 approximately doubled the number of cones in the central retina at P50 (Figure 2, C and D). In contrast, co-infection with AAV8-RedO-FLEX-CD47, a control vector with the CD47 sequence inverted, did not significantly change the number of remaining cones. To assess cone preservation with CD47 beyond the central retina, entire rdl retinas were next analyzed for GFP-positive cones using flow cytometry (Supplemental Figure 1). Consistent with the histological findings, this method showed greater cone counts at P50 with 
AAV8-RedO-GFP plus AAV8-RedO-CD47 than AAV8-RedO-GFP only (Supplemental Figure

1). To also gauge cone survival versus untreated eyes, $r d 1$ retinas at P50 were immunostained for cone arrestin. Relative to central retinas without treatment or receiving AAV8-RedO-GFP alone, addition of AAV8-RedO-CD47 again resulted in more cones as defined by this marker

(Supplemental Figure 2). Together, these data demonstrated that CD47 expression could promote survival of cones in $r d 1$ mice.

\section{CD47 promotes cone survival and retention of vision in multiple genetic models}

To determine if CD47 might similarly slow retinal degeneration in other models of RP, AAV8RedO-CD47 was tested in $r d 10$ mice, which carry a missense mutation in $P d e 6 b$, and $R h o^{-/-}$ mice, which lack rhodopsin. In both $r d 10$ retinas at $\mathrm{P} 100$ and $\mathrm{P} 130$ and $R h o^{-/}$retinas at $\mathrm{P} 150$, AAV8-RedO-CD47 again improved the number of cones (Figure 3, A-C and Supplemental Figure 1), suggesting that CD47 may generically combat cone degeneration. To investigate the therapeutic relevance of AAV8-RedO-CD47, treated animals were then subjected to two visually dependent behavioral assays. First, a light-dark discrimination test was performed by leveraging the natural preference of sighted mice for dark rather than well-illuminated spaces (see Methods). In keeping with this, wild-type animals spent $\sim 70 \%$ of their time in the dark half of a 50:50 light-dark environment, while $r d 1$ mice without treatment or receiving only AAV8-RedOGFP divided their time evenly between the two chambers (Figure 3D). Compared to these latter two groups, $r d 1$ animals receiving AAV8-RedO-GFP plus AAV8-RedO-CD47 spent significantly more time in the dark, consistent with better preservation of visual function. As a second measure of vision, mice were evaluated using an optomotor assay in which moving stripes were presented to elicit the visually dependent optomotor response. By varying the spatial 
frequency of stripes to make them easier or more difficult to see, the visual threshold in each eye could be estimated (24). In rd10 animals tested at P60, visual thresholds were significantly higher in eyes infected with AAV8-RedO-GFP plus AAV8-RedO-CD47 than AAV8-RedO-GFP alone (Figure 3E), again suggesting better retention of sight. CD47 expression thus not only promotes cone survival in different mouse models of RP, but also protects from vision loss, supporting its potential use as a mutation-agnostic therapy for this condition.

\section{Delayed expression of CD47 ameliorates cone death}

Clinically, patients with RP are often diagnosed following the development of night blindness (3). We therefore asked whether CD47 could still protect cones even after the majority of rods have died. To model this scenario, we bred R26-CreERT2 mice, which undergo inducible Cre activation in the presence of tamoxifen, with the $r d l$ strain to obtain $r d 1$;CreERT2/+ animals. When combined with a flip-excision (FLEX) vector (25), this approach allowed for delayed AAV expression while avoiding the technical challenges of subretinal injections in older mice. As a test for CreERT2-mediated recombination, $\mathrm{P} 0-1 \mathrm{rd1}$;CreERT2/+ animals were infected with AAV8-RedO-FLEX-mCherry, a vector designed to express mCherry only after exposure to tamoxifen (Figure 4A). In the absence of tamoxifen, AAV8-RedO-FLEX-mCherry produced no detectable fluorescence after 30 days (Figure 4B). However, the same vector with tamoxifen from P19-21 led to robust mCherry expression throughout the retina. Using this strategy and an analogous AAV8-RedO-FLEX-CD47 vector, the effect of delayed CD47 expression on cone survival was examined. In $r d 1$;CreERT2/+ animals co-infected with AAV8-RedO-GFP plus AAV8-RedO-FLEX-CD47 but without tamoxifen, the number of cones at P50 was comparable to that seen in $r d 1$ mice (Figure 2D and Figure 4, C and D). In contrast, in animals additionally 
receiving tamoxifen from P19-21, an age by which most rods have died (6), significantly more GFP-positive cones were observed. Collectively, these findings suggest that CD47 may alleviate cone degeneration even if administered later in the disease progression.

\section{CD47 requires SIRPa but not microglia or TSP1 to preserve cones}

In the brain, the "don't eat me" function of CD47 is thought to be largely mediated by microglia $(16,26)$. We thus suspected that AAV8-RedO-CD47 might protect cones by inhibiting their phagocytosis by microglia. To investigate this mechanism, mice infected subretinally with AAV8-RedO-CD47 had their microglia pharmacologically depleted using PLX5622, a small molecule blocking a receptor on microglia essential for their survival (27). In the retina, PLX5622 depleted $~ 99 \%$ of microglia within 15 days (Figure 5, A and B), demonstrating virtually complete elimination of this cell type. Consistent with our previous observations (10), PLX5622 administration in $r d 1$ mice from P20-49 had no significant effect on cone counts in eyes receiving only AAV8-RedO-GFP (Figure 5, C and D). Surprisingly, PLX5622 also failed to perturb cone preservation with AAV8-RedO-CD47, indicating that microglia were dispensable for its mechanism of action.

There are two major pathways by which CD47 is known to mediate intercellular signaling. Through its extracellular domain, CD47 can interact with $\operatorname{SIRP} \alpha$, a transmembrane protein expressed on a broad range of cell types including microglia, macrophages, dendritic cells, and neurons (28). In addition, CD47 can be activated by thrombospondin-1 (TSP1), a secreted protein whose binding to CD47 has been shown to help resolve subretinal inflammation (29). To determine if either TSP1-CD47 or CD47-SIRP $\alpha$ signaling were necessary for CD47 to save 
cones, we generated $r d 1$; TSP $1^{-/-}$and $r d 1$;SIRP $\alpha^{-/-}$mice, which exhibited loss of retinal TSP1 and SIRP $\alpha$, respectively (Figure 6A). In $r d 1$; TSP1 ${ }^{-/-}$animals, co-infection with AAV8-RedO-GFP plus AAV8-RedO-CD47 doubled the number of remaining cones relative to AAV8-RedO-GFP alone (Figure 6, B and C). In contrast, in $r d 1$; $\mathrm{SIRP} \alpha^{-/-}$mice, no difference in cone survival was observed with or without AAV8-RedO-CD47. These results demonstrated that the therapeutic effect of AAV8-RedO-CD47 requires SIRP $\alpha$ but not TSP1. Protection of cones with CD47 therefore likely occurs via increased CD47-SIRP $\alpha$ signaling involving one or more nonmicroglial cell types. 


\section{DISCUSSION}

Despite a growing number of gene therapy programs targeting IRDs, the vast majority of patients still lack effective treatment. Correcting individual genes or mutations may improve this situation, but only for subgroups who have those specific lesions, highlighting the need for an intervention that can benefit many more people. In this study, we developed AAV8-RedO-CD47, a novel gene therapy vector that protected cones and vision in multiple models of RP, suggesting it as a potential generic treatment for patients with different mutations. Importantly, delayed expression of CD47 until after most rods had died also preserved cones, implying that this therapy would still be beneficial if administered later in the disease progression. AAV8-RedOCD47 may thus offer a possible treatment option for many patients with RP, including those who are diagnosed at older ages or with genetics that preclude straightforward gene replacement. The vector might further help combat cone death in other IRDs and degenerative retinal disorders such as age-related macular degeneration (AMD), which affects an estimated 200 million people worldwide (30).

One key question is the mechanism by which CD47 makes cones more resistant to degeneration. While we initially hypothesized that better cone survival would be secondary to reduced engulfment by microglia, this explanation is incompatible with the PLX5622 experiments, in which near complete elimination of retinal microglia failed to perturb CD47-mediated protection of cones. Additionally, although cones may have been exposed to TSP1 secreted from the adjacent retinal pigment epithelium (RPE), AAV8-RedO-CD47 still helped preserve cones in the absence of TSP1-CD47 signaling. Instead, cone rescue with AAV8-RedO-CD47 required SIRP $\alpha$, indicating that CD47 on cones likely interacts with $\mathrm{SIRP} \alpha$ on non-microglial cells to alleviate 
degeneration. Outside of microglia, SIRP $\alpha$ in the eye is normally expressed in the synapse-rich plexiform layers of the retina (22). However, the protein is also present on multiple immune cell populations, including monocytes, macrophages, neutrophils, and dendritic cells, and has more recently been described on natural killer (NK) and a subset of cytotoxic T cells $(28,31,32)$. Binding of CD47 to SIRP $\alpha$ on immune cell populations consistently acts as a negative checkpoint, whether by inhibiting phagocytosis, preventing cell killing, or impeding recruitment of adaptive immunity (28). While Müller glia, another cell type in the retina, have also been shown to phagocytose photoreceptors (33), these cells do not appreciably express SIRP $\alpha$ (34). We therefore suspect that increased CD47-SIRP $\alpha$ signaling with AAV8-RedO-CD47 may save cones by suppressing dysregulated immune cells that would otherwise contribute to cone demise.

Therapeutic interest in the CD47-SIRP $\alpha$ axis has grown tremendously in recent years following the discovery that many cancers co-opt CD47 expression to evade anti-tumor immunity (35). Blocking CD47 and SIRP $\alpha$ have since become promising avenues to induce killing of tumor cells $(36,37)$, with several modalities now being trialed in patients $(38,39)$. Rather than disrupt, here we augmented CD47-SIRP $\alpha$ signaling in the eye by using an AAV vector to express CD47. This approach was able to slow progression in multiple models of a blinding disease and may likewise be applicable to other neurodegenerative conditions. For example, CD47 levels have been found to be low in active lesions during multiple sclerosis (40), hinting that increasing CD47 in these regions might ameliorate neuroinflammation. It would similarly be interesting to see if CD47 expression could aid other cell types that undergo degeneration such as retinal ganglion cells in glaucoma or motor neurons in amyotrophic lateral sclerosis (ALS). 


\section{METHODS}

Mice. CD-1 (\#022) and FVB (\#207) mice were purchased from Charles River Laboratories. CX3CR1 ${ }^{\mathrm{GFP}}(\# 005582), r d 10$ (\#004297), C3H (\#000659), sighted C3H (\#003648), R26CreERT2 (\#008463), and TSP1 ${ }^{-/-}$(\#006141) mice were purchased from The Jackson Laboratory. $R h o^{-/-}$mice were a gift from Janis Lem (Tufts University) (41). SIRPa ${ }^{-/-}$mice were a gift from Beth Stevens (Harvard Medical School) (16). CX3CR1 GFP , R26-CreERT2, TSP1 ${ }^{-/-}$, and SIRP $\alpha^{-/-}$ lines were crossed with FVB mice for at least four generations to obtain the following strains: sighted CX3CR $1^{\mathrm{GFP} /+}, r d 1 ; \mathrm{CX} 3 \mathrm{CR} 1^{\mathrm{GFP} /+}, r d 1$; $\mathrm{CreERT} 2 /+, r d 1 ; \mathrm{TSP}^{+/-}, r d 1 ; \mathrm{TSP}^{-/-}, r d 1 ; \mathrm{SIRP} \alpha^{+/-}$ , and $r d 1$; $\mathrm{SIRP} \alpha^{-/}$. Genotyping was performed by Transnetyx using real-time PCR. Mice were maintained at Harvard Medical School on a 12-hour alternating light and dark cycle. Animals were housed in standard ventilated racks at a density of up to five per cage. Both males and females were used in all experiments and were randomly assigned to experimental groups.

Histology. Retinal flat-mounts from $r d l$ (FVB), $r d 10$, and $R h o^{-/-}$mice were prepared as previously described $(9,10)$. Following dissection of enucleated eyes in phosphate-buffered saline (PBS), retinas were fixed in $4 \%$ paraformaldehyde for 30 minutes at room temperature, washed twice with PBS, and relaxed with four radial incisions. For cone arrestin immunostaining, retinas were additionally blocked with 5\% donkey serum and $0.3 \%$ Triton X100 in PBS for one hour at room temperature, incubated with 1:3000 of anti-cone arrestin (Millipore) in blocking solution overnight at $4^{\circ} \mathrm{C}$, and labeled with 1:1000 of donkey anti-rabbit secondary (Jackson ImmunoResearch) in PBS for two hours at room temperature. Retinas were mounted on microscope slides using Fluoromount-G (SouthernBiotech) with the ganglion cell layer facing up. For retinal cross-sections, enucleated eyes were dissected to remove the cornea, 
iris, lens, and ciliary body. The remaining eye cups were cryoprotected in a sucrose gradient, frozen in a 1:1 mixture of optimal cutting temperature compound (Tissue-Tek) and 30\% sucrose in PBS, and sectioned on a Leica CM3050S cryostat (Leica Microsystems) at a thickness of 20 $\mu \mathrm{m}$. Tissues were blocked with 5\% donkey serum and 0.1\% Triton X-100 in PBS for one hour at room temperature and incubated with 1:1000 of anti-CD47 (BD Biosciences), 1:500 of antiTSP1 (Abcam), or 1:500 of anti-SIRP $\alpha$ (QED Bioscience) in blocking solution overnight at $4^{\circ} \mathrm{C}$. Sections were subsequently labeled with 1:1000 of the appropriate secondary antibody (Jackson ImmunoResearch) in PBS for two hours at room temperature followed by $0.5 \mu \mathrm{g} / \mathrm{mL}$ of $4^{\prime}, 6$ diamidino-2-phenylindole (DAPI) (ThermoFisher Scientific) in PBS for five minutes at room temperature prior to mounting.

Ex vivo phagocytosis assay. Freshly dissected retinas were incubated with gentle agitation for one hour at $37^{\circ} \mathrm{C}$ in $1 \mathrm{mg} / \mathrm{mL}$ of pHrodo Red-conjugated zymosan bioparticles (ThermoFisher Scientific) resuspended in retinal culture media (1:1 mixture of DMEM and F-12 supplemented with L-glutamine, B27, N2, and penicillin-streptomycin). Retinas were subsequently washed with PBS and dissociated using cysteine-activated papain as previously described (9). After two additional washes, samples were passed through a $40 \mu \mathrm{m}$ filter and stained with $0.5 \mu \mathrm{g} / \mathrm{mL}$ of DAPI in FACS buffer (2 mM EDTA and 2\% fetal bovine serum in PBS) to exclude non-viable cells. Data were collected on a Cytek DxP11 cytometer and analyzed using FlowJo 10. CX3CR1-positive microglia were defined as phagocytic if positive for pHrodo Red.

AAV vector cloning and production. The AAV-human red opsin-GFP-WPRE-bGH (AAV8RedO-GFP) plasmid was a gift from Botond Roska (Institute for Molecular and Clinical 
Ophthalmology Basel) (42). The AAV8-RedO-CD47 plasmid was cloned by replacing the GFP coding sequence in AAV8-RedO-GFP with that of the most abundant isoform of mouse CD47 (NM_010581.3) (43). The AAV8-RedO-FLEX-mCherry plasmid was cloned by replacing the GFP coding sequence in AAV8-RedO-GFP with that of mCherry inverted and flanked by lox2272 and loxP sites. The AAV8-RedO-FLEX-CD47 plasmid was cloned by replacing the inverted mCherry sequence in AAV8-RedO-FLEX-mCherry with that of inverted CD47. AAV vectors were generated as previously described by transfecting $293 \mathrm{~T}$ cells with a mixture the vector plasmid, adenovirus helper plasmid, and rep2/cap8 packaging plasmid (44, 45). Seventytwo hours post-transfection, viral particles were harvested from the supernatant, PEGylated overnight, precipitated by centrifugation, treated with Benzonase nuclease (Sigma-Aldrich), and purified through an iodixanol gradient before collection in 100-200 $\mu \mathrm{L}$ of PBS. Vectors were semi-quantitatively titered by SYPRO Ruby (Molecular Probes) for viral capsid proteins (VP1, VP2, and VP3) relative to a reference vector titered by real-time PCR

$A A V$ vector delivery. Subretinal injections of AAV vectors were performed on neonatal mice (P0-1) as previously described (46). Following anesthetization of the animal on ice, the palpebral fissure was gently opened with a 30-gauge needle and the eye exposed. Using a glass needle controlled by a FemtoJet microinjector (Eppendorf), $\sim 0.25 \mu \mathrm{L}$ of vectors were then delivered into the subretinal space. Both left and right eyes were used for injections. AAV8-RedO-GFP was administered at $\sim 5 \times 10^{8}$ vector genomes (vg) per eye, a dose capable of transducing up to $99 \%$ of cones (4). All other vectors were administered at $\sim 1 \times 10^{9} \mathrm{vg}$ per eye. 
Image acquisition and analysis. Retinal flat-mounts were imaged using a Nikon Ti inverted widefield microscope (10x or 20x air objective). Retinal cross-sections were imaged using a Zeiss LSM710 scanning confocal microscope (20x air objective or 40x oil objective). All image analysis was performed using ImageJ. To quantify GFP-positive or cone arrestin-positive cones, custom ImageJ modules were used as previously described $(9,10)$. For each flat-mount, the locations of the optic nerve head and four retinal leaflets were first manually defined. The number of GFP-positive or cone arrestin-positive objects within the region corresponding to the central retina was then automatically counted and used to represent the number of cones in that sample.

Light-dark test. Light avoidance in $r d l(\mathrm{C} 3 \mathrm{H})$ and sighted $\mathrm{C} 3 \mathrm{H}$ mice following no treatment or treatment in both eyes was assessed as previously described (10). Testing was not performed in rd10 animals as light avoidance in this strain is preserved for at least four months (data not shown). A plastic chamber (Med Associates) measuring $28 \mathrm{~cm}$ (length) by $28 \mathrm{~cm}$ (width) by 21 $\mathrm{cm}$ (height) was divided into two equally sized compartments: one dark and one brightly illuminated ( 900 lux). The two compartments were connected by a small opening and differed in temperature by less than $1^{\circ} \mathrm{C}$. At the start of each trial, a mouse was placed in the illuminated compartment and its activity recorded for ten minutes. If after one minute, the animal had not yet entered the dark compartment, it was gently guided there, removed from the chamber, and the trial restarted. Mice were tracked using infrared sensors and location data analyzed with Activity Monitor (Med Associates). Percent time spent in dark was calculated based on the final nine minutes of each trial. 
Optomotor assay. Optomotor responses are absent in the $r d 1$ strain (47) and were consequently assessed in $r d 10$ mice. Visual thresholds were measured by an observer (Y.X.) blinded to the treatment groups using the OptoMotry System (CerebralMechanics) as previously described (9). Animals were placed in a chamber with bright background luminance to saturate rods and presented with moving gratings of varying spatial frequencies. Left and right eyes were assessed using clockwise and counterclockwise gratings, respectively, as the optomotor response is evoked by temporal-to-nasal motion in mice (24). For each eye, the highest spatial frequency at which the mouse tracked the grating was determined to be the visual threshold.

Tamoxifen injections. Tamoxifen (Sigma-Aldrich) was dissolved in corn oil (Sigma-Aldrich) and dosed at $2 \mathrm{mg}$ daily from P19-21 via intraperitoneal injections.

Microglia depletion. Microglia were depleted using PLX5622 (Plexxikon), an orally available CSF1R inhibitor. PLX5622 was formulated into AIN-76A rodent chow (Research Diets) at 1200 $\mathrm{mg} / \mathrm{kg}$ and provided ad libitum during periods of depletion. For quantification of retinal microglia, freshly dissected retinas were dissociated using cysteine-activated papain as previously described (9). Cells were subsequently blocked with 1:100 of anti-CD16/32 (BD Pharmingen) for five minutes on ice followed by incubation with 1:200 of PE-Cy5-conjugated anti-CD11b (BioLegend), 1:200 of APC-Cy7-conjugated anti-Ly6C (BioLegend), and 1:200 of APC-Cy7conjugated anti-Ly6G (BioLegend) for 20 minutes on ice. After washes, samples were passed through a $40 \mu \mathrm{m}$ filter and stained with $0.5 \mu \mathrm{g} / \mathrm{mL}$ of DAPI in FACS buffer. Data were collected on a BD FACSAria II and analyzed using FlowJo 10. 
Statistics. Statistical analyses were performed using GraphPad Prism software. Experimental groups were compared using two-tailed Student's t-tests, with the addition of a Bonferroni correction if three or more comparisons were performed. A $P$ value of less than 0.05 was considered statistically significant. Information on group data and replicates is reported in each figure legend.

Study approval. All animal experiments were conducted under protocols approved by the Institutional Animal Care and Use Committee (IACUC) of Harvard Medical School. 
bioRxiv preprint doi: https://doi.org/10.1101/2021.04.23.440841; this version posted April 23, 2021. The copyright holder for this preprint (which

was not certified by peer review) is the author/funder, who has granted bioRxiv a license to display the preprint in perpetuity. It is made available under aCC-BY-NC-ND 4.0 International license.

\section{AUTHOR CONTRIBUTIONS}

S.K.W. and C.L.C. designed the study. S.K.W. and Y.X. performed the experiments and analyzed the data. S.K.W. and C.L.C. wrote the manuscript with input from Y.X. 


\section{ACKNOWLEDGEMENTS}

The authors thank Sophia Zhao, the Flow Cytometry Core at Joslin Diabetes Center, the Mouse Behavior Core at Harvard Medical School, and Microscopy Resources on the North Quad at

Harvard Medical School for discussions and technical support. This work was supported by the Howard Hughes Medical Institute (C.L.C.) and VitreoRetinal Surgery Foundation (S.K.W.). The authors declare no competing interests. 


\section{REFERENCES}

1. Takahashi V et al. Gene therapy in inherited retinal degenerative diseases, a review.

Ophthalmic Genet. 2018;39(5).

2. Russell S et al. Efficacy and safety of voretigene neparvovec (AAV2-hRPE65v2) in patients with RPE65-mediated inherited retinal dystrophy: a randomised, controlled, open-label, phase 3 trial. Lancet. 2017;390(10097):849-860.

3. Hartong DT, Berson EL, Dryja TP. Retinitis pigmentosa. Lancet. 2006;368(9549):1795-1809.

4. Xiong W et al. NRF2 promotes neuronal survival in neurodegeneration and acute nerve damage. J Clin Invest. 2015;125(4):1433-1445.

5. Komeima $\mathrm{K}$ et al. Antioxidants reduce cone cell death in a model of retinitis pigmentosa. Proc Natl Acad Sci U S A. 2006;103(30):11300-5.

6. Punzo C, Kornacker K, Cepko CL. Stimulation of the insulin/mTOR pathway delays cone death in a mouse model of retinitis pigmentosa. Nat Neurosci. 2009;12(1):44-52.

7. Aït-Ali N et al. Rod-Derived Cone Viability Factor Promotes Cone Survival by Stimulating Aerobic Glycolysis. Cell. 2015;161(4):817-832.

8. Xue $\mathrm{Y}$ et al. AAV-Txnip prolongs cone survival and vision in mouse models of retinitis pigmentosa. Elife. 2021;10.

9. Wang SK et al. Soluble CX3CL1 gene therapy improves cone survival and function in mouse models of retinitis pigmentosa. Proc Natl Acad Sci U S A. 2019;116(20):10140-10149.

10. Wang SK, Xue Y, Cepko CL. Microglia modulation by TGF- $\beta 1$ protects cones in mouse models of retinal degeneration. J Clin Invest. 2020;140(8):4360-4369.

11. Gupta N, Brown KE, Milam AH. Activated microglia in human retinitis pigmentosa, lateonset retinal degeneration, and age-related macular degeneration. Exp Eye Res. 2003;76(4):463- 
71.

12. Subhramanyam CS et al. Microglia-mediated neuroinflammation in neurodegenerative diseases. Semin Cell Dev Biol. 2019;94:112-120.

13. Zhao L et al. Microglial phagocytosis of living photoreceptors contributes to inherited retinal degeneration. EMBO Mol Med. 2015;7(9):1179-1197.

14. Peng B et al. Suppression of Microglial Activation Is Neuroprotective in a Mouse Model of Human Retinitis Pigmentosa. J Neurosci. 2014;34(24):8139-8150.

15. Oldenborg PA, Gresham HD, Lindberg FP. CD47-signal regulatory protein $\alpha$ (SIRP $\alpha)$ regulates Fc $\gamma$ and complement receptor-mediated phagocytosis. J Exp Med. 2001;193(7):855861.

16. Lehrman EK et al. CD47 Protects Synapses from Excess Microglia-Mediated Pruning during Development. Neuron. 2018;100(1):120-134.e6.

17. Chang B et al. Retinal degeneration mutants in the mouse. Vision Res. 2002;42(4):517-525. 18. Jung S et al. Analysis of fractalkine receptor CX(3)CR1 function by targeted deletion and green fluorescent protein reporter gene insertion. Mol Cell Biol. 2000;20(11):4106-14.

19. O'Koren EG et al. Microglial Function Is Distinct in Different Anatomical Locations during Retinal Homeostasis and Degeneration. Immunity. 2019;50(3):723-737.e7.

20. Miksa M et al. A novel method to determine the engulfment of apoptotic cells by macrophages using pHrodo succinimidyl ester. J Immunol Methods. 2009;342(1-2):71-77.

21. Park SY, Kim IS. Engulfment signals and the phagocytic machinery for apoptotic cell clearance. Exp Mol Med. 2017;49(5).

22. Mi ZP et al. Expression of a synapse-associated membrane protein, P84/SHPS-1, and its ligand, IAP/CD47, in mouse retina. J Comp Neurol. 2000;416(3):335-344. 
23. Wu DM et al. Nrf2 overexpression rescues the RPE in mouse models of retinitis pigmentosa. JCI Insight. 2021;6(2).

24. Douglas RM et al. Independent visual threshold measurements in the two eyes of freely moving rats and mice using a virtual-reality optokinetic system. Vis Neurosci. 2005;22(5):677684.

25. Atasoy D et al. A FLEX switch targets channelrhodopsin-2 to multiple cell types for imaging and long-range circuit mapping. $J$ Neurosci. 2008;28(28):7025-7030.

26. Hutter $\mathrm{G}$ et al. Microglia are effector cells of CD47-SIRP $\alpha$ antiphagocytic axis disruption against glioblastoma. Proc Natl Acad Sci U S A. 2019;116(3):997-1006.

27. Elmore MRP et al. Colony-stimulating factor 1 receptor signaling is necessary for microglia viability, unmasking a microglia progenitor cell in the adult brain. Neuron. 2014;82(2):380-97.

28. Matlung HL et al. The CD47-SIRP $\alpha$ signaling axis as an innate immune checkpoint in cancer. Immunol Rev. 2017;276(1):145-164.

29. Calippe B et al. Complement Factor H Inhibits CD47-Mediated Resolution of Inflammation. Immunity. 2017;46(2):261-272.

30. Wong WL et al. Global prevalence of age-related macular degeneration and disease burden projection for 2020 and 2040: A systematic review and meta-analysis. Lancet Glob Heal. 2014;2(2).

31. Deuse T et al. The SIRP $\alpha-C D 47$ immune checkpoint in NK cells. J Exp Med. 2021;218(3). 32. Myers LM et al. A functional subset of CD8 + T cells during chronic exhaustion is defined by SIRP $\alpha$ expression. Nat Commun. 2019;10(1).

33. Sakami S, Imanishi Y, Palczewski K. Müller glia phagocytose dead photoreceptor cells in a mouse model of retinal degenerative disease. FASEB J. 2019;33(3):3680-3692. 
34. Shekhar K et al. Comprehensive Classification of Retinal Bipolar Neurons by Single-Cell Transcriptomics. Cell. 2016;166(5):1308-1323.e30.

35. Chao MP, Majeti R, Weissman IL. Programmed cell removal: A new obstacle in the road to developing cancer. Nat Rev Cancer. 2012;12(1):58-67.

36. Willingham SB et al. The CD47-signal regulatory protein alpha (SIRPa) interaction is a therapeutic target for human solid tumors. Proc Natl Acad Sci U S A. 2012;109(17):6662-6667. 37. Weiskopf $\mathrm{K}$ et al. Engineered SIRP $\alpha$ variants as immunotherapeutic adjuvants to anticancer antibodies. Science. 2013;341(6141):88-91.

38. Sikic BI et al. First-in-human, first-in-class phase i trial of the anti-CD47 antibody Hu5F9G4 in patients with advanced cancers. J Clin Oncol. 2019;37(12):946-953.

39. Advani R et al. CD47 Blockade by Hu5F9-G4 and Rituximab in Non-Hodgkin's Lymphoma. N Engl J Med. 2018;379(18):1711-1721.

40. Han MH et al. Janus-like opposing roles of CD47 in autoimmune brain inflammation in humans and mice. J Exp Med. 2012;209(7):1325-1334.

41. Lem J et al. Morphological, physiological, and biochemical changes in rhodopsin knockout mice. Proc Natl Acad Sci U S A. 1999;96(2):736-741.

42. Busskamp V et al. Genetic Reactivation of Cone Photoreceptors Restores Visual Responses in Retinitis Pigmentosa. Science. 2010;329(5990):413-417.

43. Brown EJ, Frazier WA. Integrin-associated protein (CD47) and its ligands. Trends Cell Biol. 2001;11(3):130-135.

44. Grieger JC, Choi VW, Samulski RJ. Production and characterization of adeno-associated viral vectors. Nat Protoc. 2006;1(3):1412-1428.

45. Xiong W et al. AAV cis-regulatory sequences are correlated with ocular toxicity. Proc Natl 
bioRxiv preprint doi: https://doi.org/10.1101/2021.04.23.440841; this version posted April 23, 2021. The copyright holder for this preprint (which

was not certified by peer review) is the author/funder, who has granted bioRxiv a license to display the preprint in perpetuity. It is made available under aCC-BY-NC-ND 4.0 International license.

Acad Sci U S A. 2019;116(12):5785-5794.

46. Wang SK et al. In Situ Detection of Adeno-associated Viral Vector Genomes with SABER-

FISH. Mol Ther Methods Clin Dev. 2020;19:376-386.

47. Abdeljalil J et al. The optomotor response: A robust first-line visual screening method for mice. Vision Res. 2005;45(11):1439-1446. 


\section{FIGURES AND FIGURE LEGENDS}

A

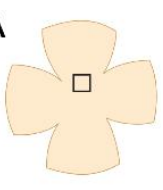

ㄱ.

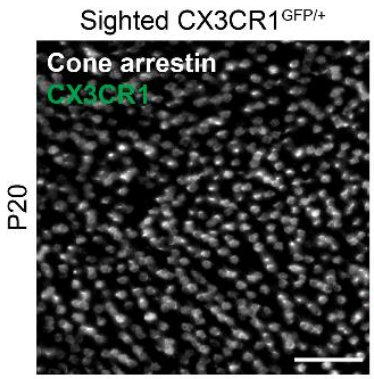

rd1;CX3CR1 ${ }^{\mathrm{GFP} /+}$

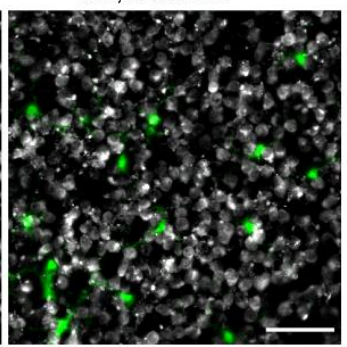

Sighted CX3CR1 1 GFP/+

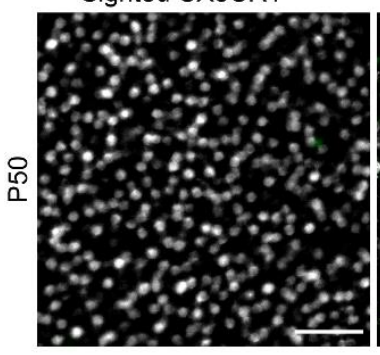

rd1;CX3CR1 $1^{\mathrm{GFP} /+}$

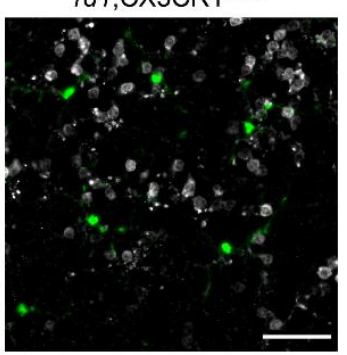

B

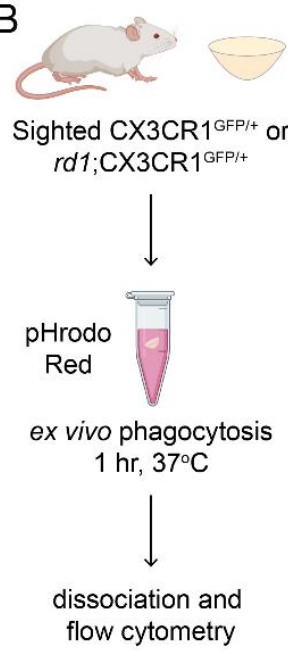

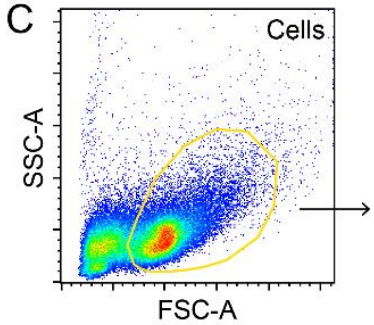

FSC-A

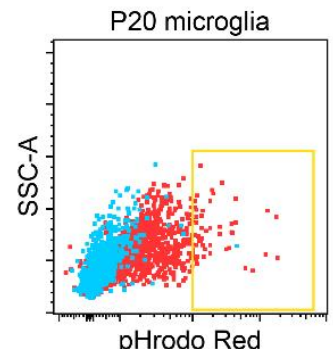

pHrodo Red

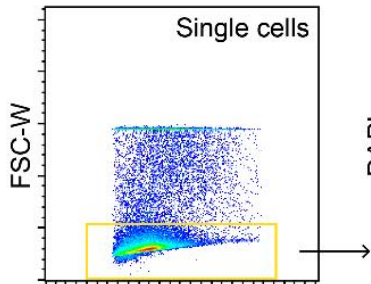

FSC-A

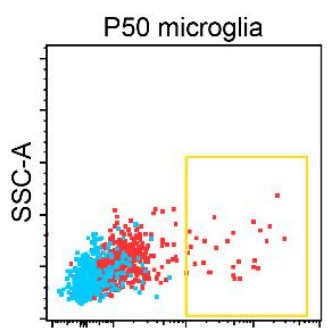

pHrodo Red
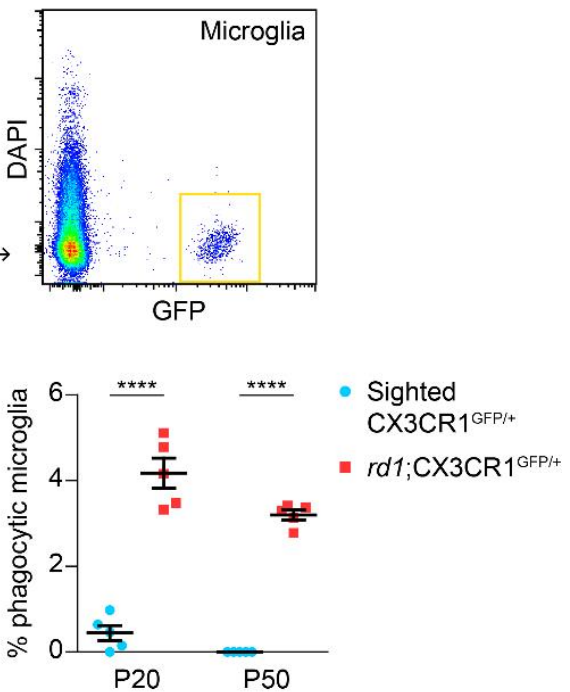

Figure 1. Cone degeneration is associated with increased microglial phagocytosis.

(A) Cone arrestin immunostaining and CX3CR1-positive microglia in flat-mounted retinas from sighted CX3CR $1^{\mathrm{GFP} /+}$ ( $r d 1$ heterozygous) and $r d 1$;CX3CR $1^{\mathrm{GFP} /+}$ mice at P20 and P50. Images were acquired from the central retina. Scale bars, $50 \mu \mathrm{m}$. (B) Schematic of ex vivo phagocytosis assay. Retinas from sighted $\mathrm{CX} 3 \mathrm{CR} 1^{\mathrm{GFP} /+}$ and $r d 1 ; \mathrm{CX} 3 \mathrm{CR} 1^{\mathrm{GFP} /+}$ mice were incubated with yeast (zymosan) particles conjugated to pHrodo Red, a pH-sensitive dye that fluoresces upon lysosomal acidification. Microglia were subsequently analyzed by flow cytometry. (C) Flow cytometry gating for microglia and quantification of microglial phagocytosis in sighted $\mathrm{CX} 3 \mathrm{CR} 1^{\mathrm{GFP} /+}(n=5)$ and $r d 1$; $\mathrm{CX} 3 \mathrm{CR} 1^{\mathrm{GFP} /+}(n=5)$ retinas at P20 and P50. Data are shown as mean \pm SEM. $* * * * ~ P<0.0001$ by two-tailed Student's t-test. 
A AAV8-RedO-GFP

$\frac{\text { human red opsin promoter - GFP }- \text { WPRE -bGH pA- }}{\text { AAV8-RedO-CD47 }} 3.6 \mathrm{~kb}-$

AAV8-RedO-CD47

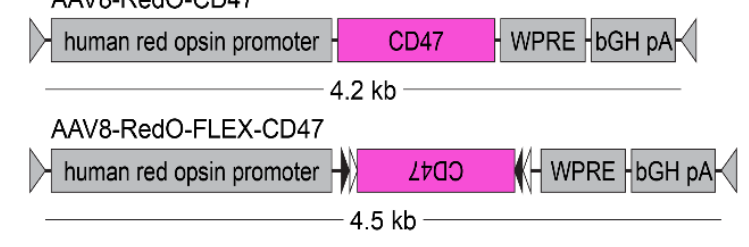

C

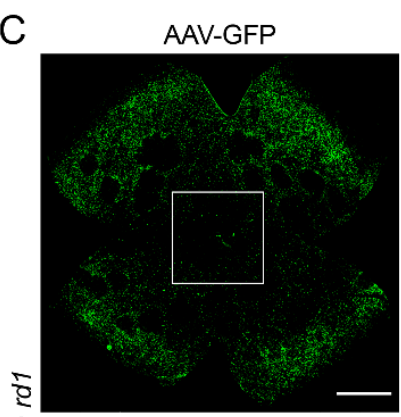

웅

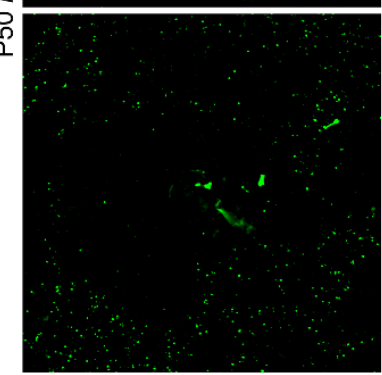

AAV-GFP + AAV-CD47
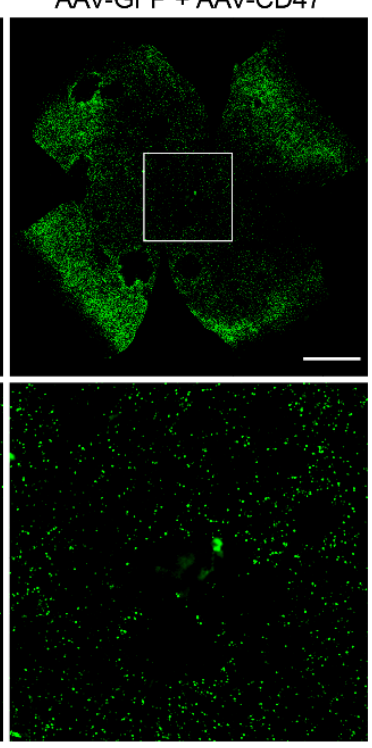

B

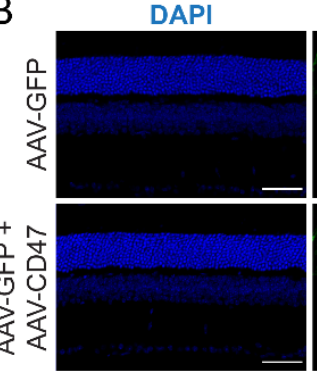

AAV-GFP + AAV-FLEX-CD47
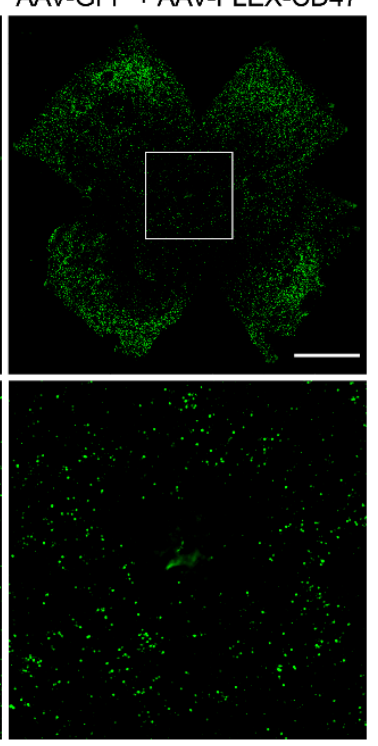

GFP
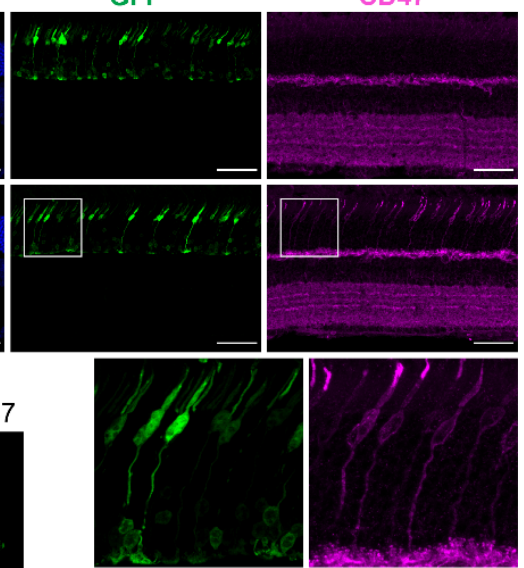

D * AAV-GFP

- AAV-GFP + AAV-CD47

- AAV-GFP + AAV-FLEX-CD47

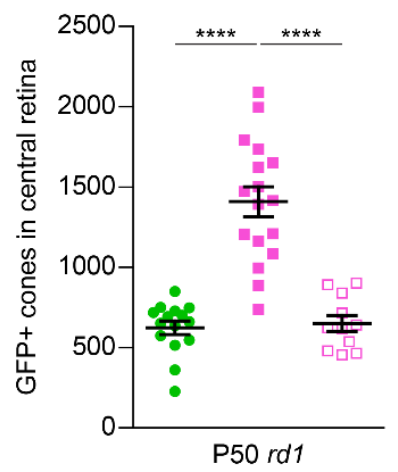

Figure 2. Effect of CD47 expression on cone survival.

(A) Schematics of AAV vectors. AAV8-RedO-FLEX-CD47 is a flip-excision (FLEX) vector in which the CD47 transgene is inverted and flanked by lox2272 (black triangles) and loxP (white triangles) sites. (B) Immunostaining for CD47 in P40 wild-type (CD-1) retinas following infection with AAV8-RedO-GFP or AAV8-RedO-GFP plus AAV8-RedO-CD47. Nuclei were labeled with 4',6-diamidino-2-phenylindole (DAPI). Scale bars, $50 \mu \mathrm{m}$. (C) Representative flatmounts of P50 rdl retinas following infection with AAV8-RedO-GFP, AAV8-RedO-GFP plus AAV8-RedO-CD47, or AAV8-RedO-GFP plus AAV8-RedO-FLEX-CD47. Paired images depict low and high magnifications. Scale bars, $1 \mathrm{~mm}$. (D) Quantification of GFP-positive cones in central retinas of $r d 1$ mice $(n=11-17)$ following infection with AAV8-RedO-GFP, AAV8RedO-GFP plus AAV8-RedO-CD47, or AAV8-RedO-GFP plus AAV8-FLEX-RedO-CD47. Data are shown as mean \pm SEM. $* * * * P<0.0001$ by two-tailed Student's t-test. 
A

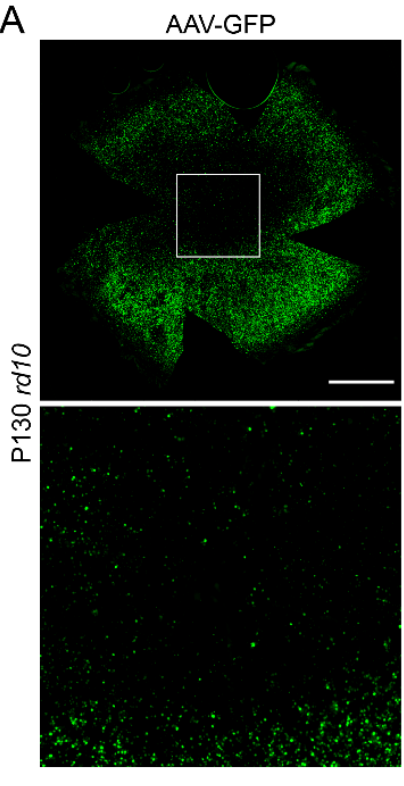

B

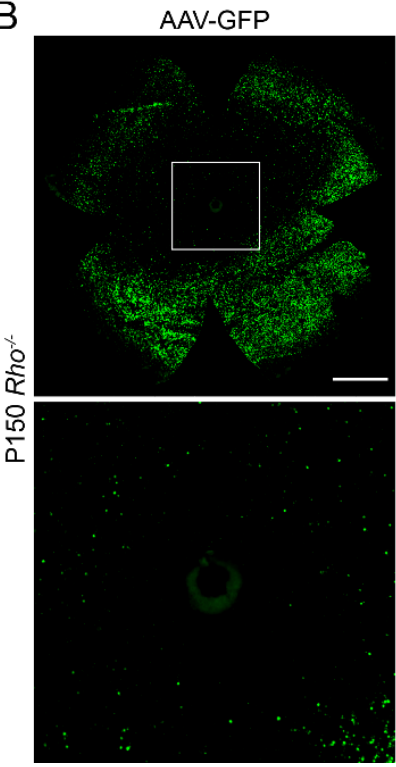

AAV-GFP + AAV-CD47

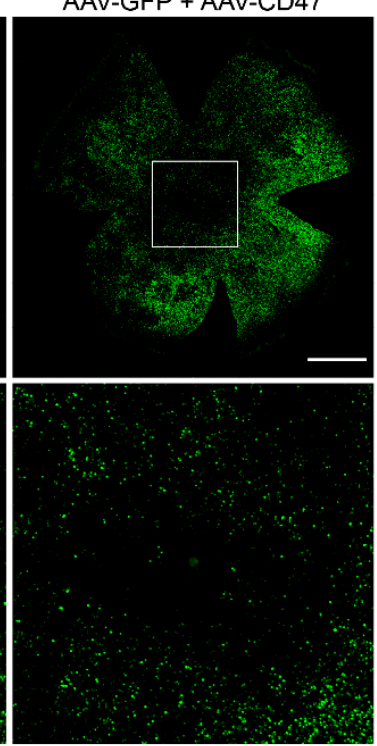

AAV-GFP + AAV-CD47

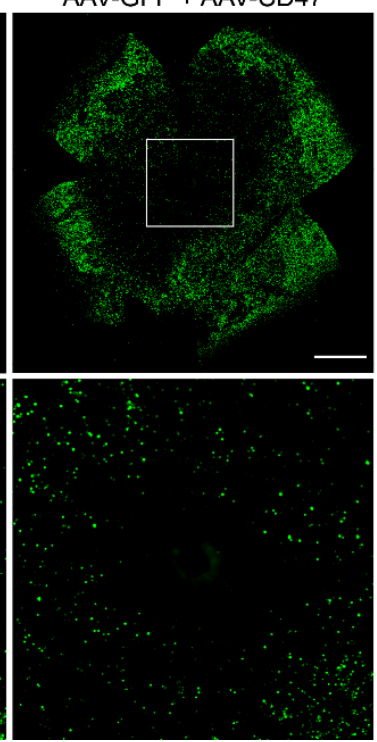

C - AAV-GFP

- AAV-GFP + AAV-CD47

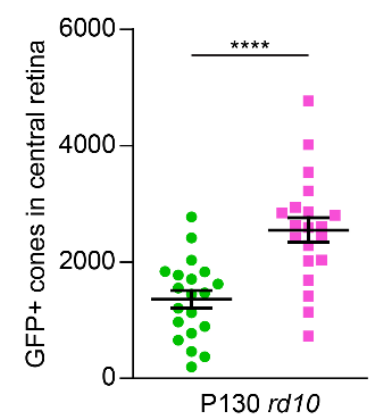

$\mathrm{P} 130 \mathrm{rd10}$

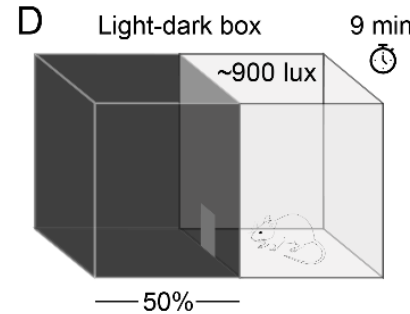

P30 sighted | $\circ$ No treatment

P30 rd1 - No treatment

- AAV-GFP

- AAV-GFP + AAV-CD47
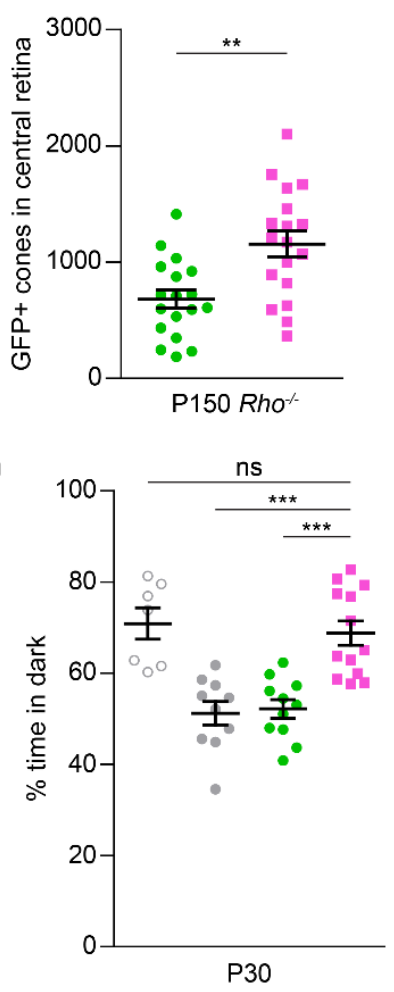

E Optomotor response

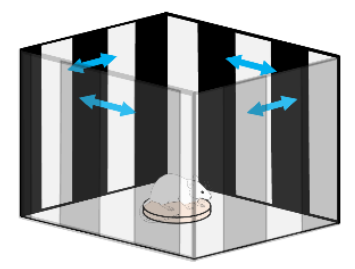

- AAV-GFP

AAV-GFP + AAV-CD47

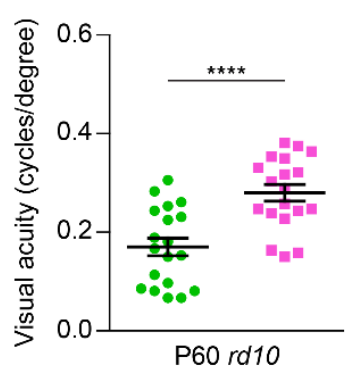

Figure 3. Effect of CD47 expression on long-term cone survival and visual function.

(A) Representative flat-mounts of P130 rd10 retinas following infection with AAV8-RedO-GFP or AAV8-RedO-GFP plus AAV8-RedO-CD47. Paired images depict low and high magnifications. Scale bars, $1 \mathrm{~mm}$. (B) Representative flat-mounts of P150 Rho ${ }^{-/}$retinas following infection with AAV8-RedO-GFP or AAV8-RedO-GFP plus AAV8-RedO-CD47. Paired images depict low and high magnifications. Scale bars, $1 \mathrm{~mm}$. (C) Quantification of GFPpositive cones in central retinas of $r d 10(n=20)$ and $R_{h o}^{-/-}(n=18)$ mice following infection with AAV8-RedO-GFP or AAV8-RedO-GFP plus AAV8-RedO-CD47. (D) Percent time spent in dark in a 50:50 light-dark box for untreated $(n=7-10)$ and $r d l(n=11-13)$ mice following infection with AAV8-RedO-GFP or AAV8-RedO-GFP plus AAV8-RedO-CD47. (E) Visual thresholds in eyes from P60 rd10 mice $(n=19)$ as measured by optomotor following infection with AAV8-RedO-GFP or AAV8-RedO-GFP plus AAV8-RedO-CD47. Data are shown as mean \pm SEM. $* * P<0.01, * * * P<0.001, * * * * P<0.0001$ by two-tailed Student's t-test for $(\mathrm{C})$ and $(\mathrm{E})$, two-tailed Student's t-test with Bonferroni correction for (D). ns, not significant. 


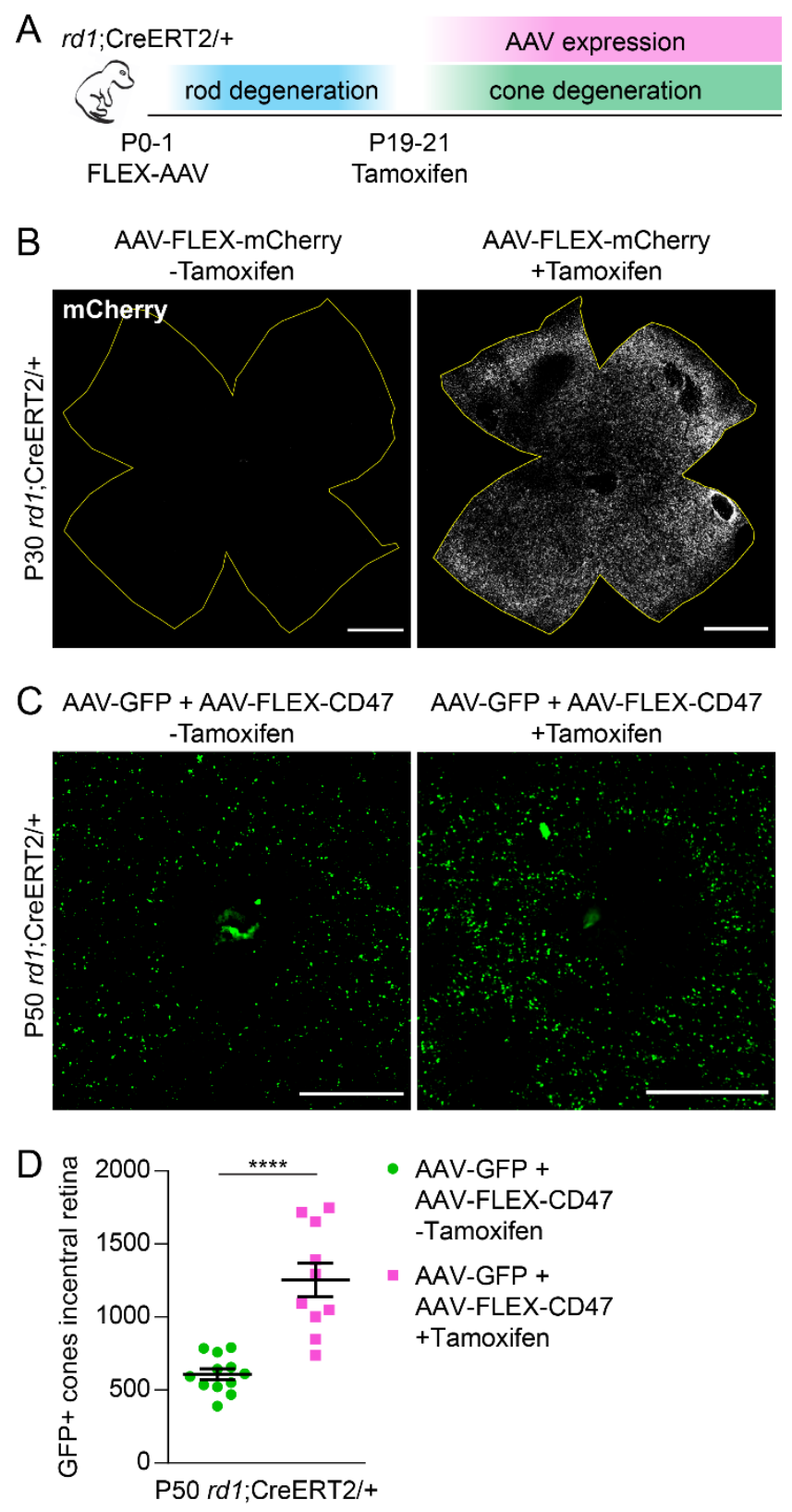

\section{Figure 4. Effect of delayed CD47 expression on cone survival.}

(A) Schematic of delayed AAV expression experiments. P0-1 rd1;CreERT2/+ mice were subretinally injected with FLEX vectors, which were subsequently activated by intraperitoneal (IP) injections of tamoxifen from P19-21. (B) Representative flat-mounts of P30 rd1;CreERT2/+ retinas following infection with AAV8-RedO-FLEX-mCherry with or without IP tamoxifen. Boundaries of each retina are depicted in yellow. Scale bars, $1 \mathrm{~mm}$. (C) Representative images of central retinas from P50 rd1; CreERT2/+ mice following infection with AAV8-RedO-GFP plus AAV8-RedO-FLEX-CD47 with or without IP tamoxifen. Scale bars, $500 \mu \mathrm{m}$. (D) Quantification of GFP-positive cones in central retinas of $r d 1$; CreERT2/+ mice $(n=10-12)$ following infection with AAV8-RedO-GFP plus AAV8-RedO-FLEX-CD47 with or without IP tamoxifen. Data are shown as mean \pm SEM. $* * * * P<0.0001$ by two-tailed Student's t-test. 

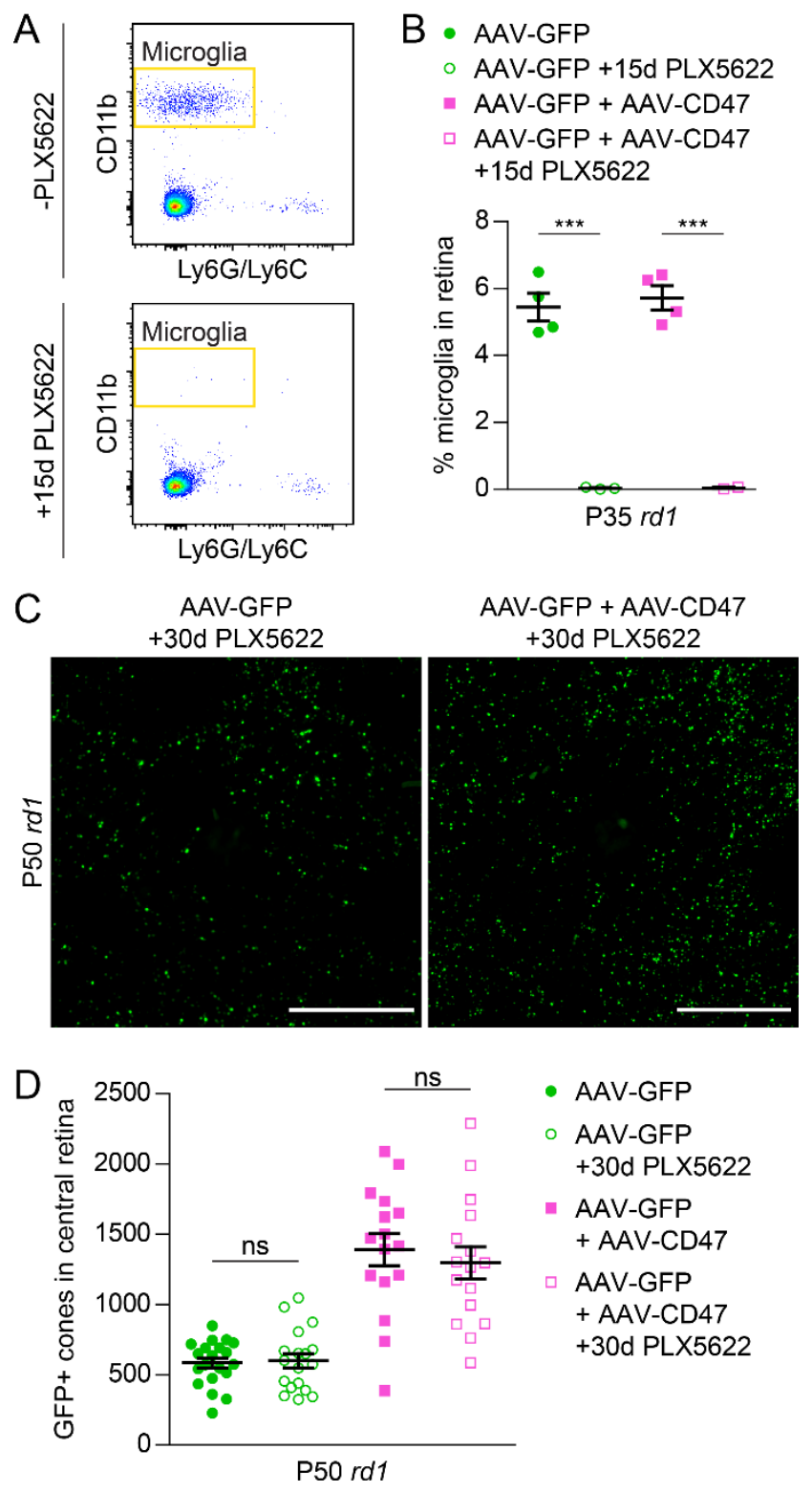

Figure 5. Role of microglia in CD47-mediated cone survival.

(A) Flow cytometry gating for retinal microglia from P35 rd1 mice with or without PLX5622 from P20-34. Microglia were defined as CD11b-positive Ly6G/Ly6C-negative cells. (B) Quantification by flow cytometry of retinal microglia from P35 rdl mice $(n=2-4)$ following infection with AAV8-RedO-GFP or AAV8-RedO-GFP plus AAV8-RedO-CD47 with or without PLX5622 from P20-34. (C) Representative images of central retinas from P50 rd1 mice following infection with AAV8-RedO-GFP or AAV8-RedO-GFP plus AAV8-RedO-CD47 and PLX5622 from P20-49. Scale bars, $500 \mu \mathrm{m}$. (D) Quantification of GFP-positive cones in central retinas of $r d 1$ mice ( $n=16$-18) following infection with AAV8-RedO-GFP or AAV8-RedO-GFP plus AAV8-RedO-CD47 and PLX5622 from P20-49. Groups without PLX5622 are taken from Figure 2D. Data are shown as mean \pm SEM. $* * * ~ P<0.001$ by two-tailed Student's t-test. ns, not significant. 


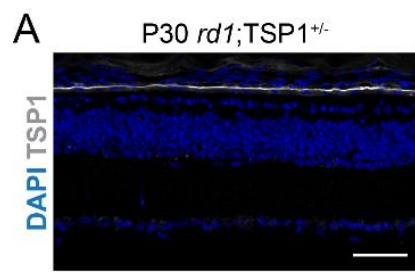

P30 rd1; SIRP $\alpha^{+1-}$

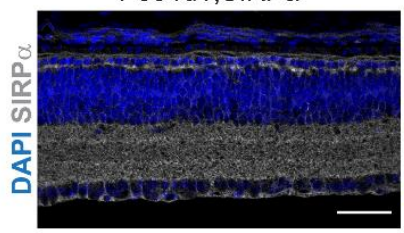

C

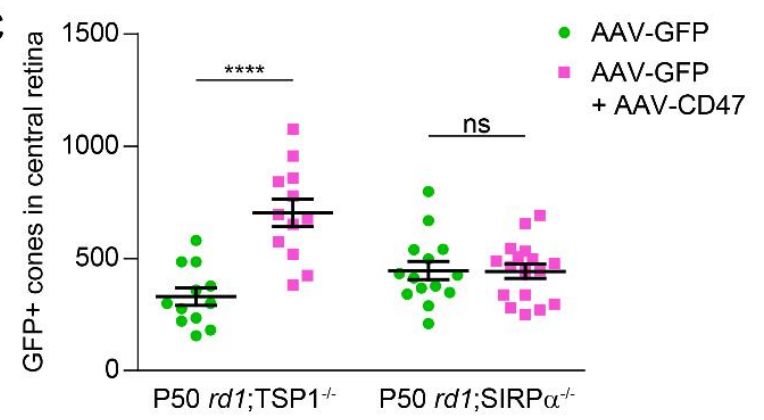

P30 rd1;TSP $1 \%$

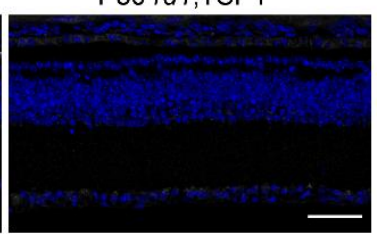

P30 rd1; SIRP $\alpha^{-1-}$

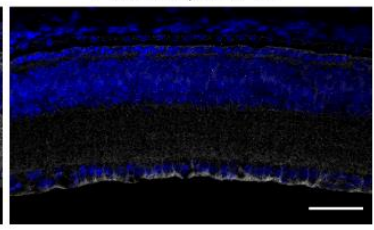

AAV-GFP

AAV-GFP
B
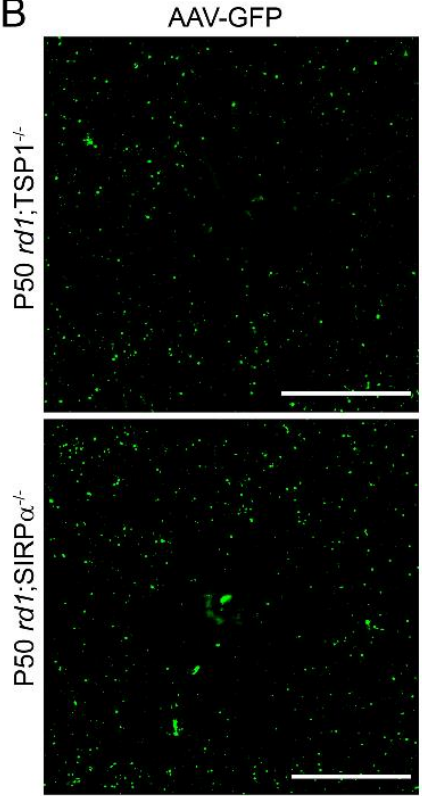

AAV-GFP + AAV-CD47

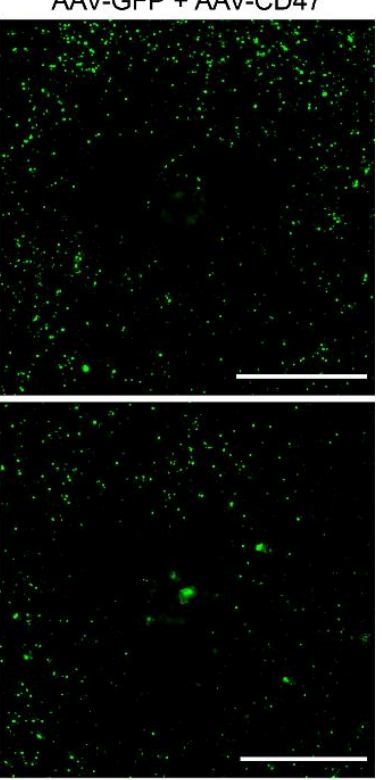

\section{Figure 6. Role of TSP1 and SIRP $\alpha$ in CD47-mediated cone survival.}

(A) Immunostaining for TSP1 and SIRP $\alpha$, respectively, in P30 rd1; TSP1 $1^{-/-}$and $r d 1$; $\mathrm{SIRP}^{-/-}$ retinas or heterozygous controls. Scale bars, $50 \mu \mathrm{m}$. (B) Representative images of central retinas from P50 rd1; $\mathrm{TSP}^{-/-}$and $r d 1$; $\mathrm{SIRP} \alpha^{-/-}$mice following infection with AAV8-RedO-GFP or AAV8-RedO-GFP plus AAV8-RedO-CD47. Scale bars, $500 \mu \mathrm{m}$. (C) Quantification of GFPpositive cones in central retinas of $r d 1 ; \mathrm{TSP}^{-/-}(n=12)$ and $r d l ; \mathrm{SIRP}^{-/-}(n=14-17)$ mice following infection with AAV8-RedO-GFP or AAV8-RedO-GFP plus AAV8-RedO-CD47. Data are shown as mean \pm SEM. $* * * * P<0.0001$ by two-tailed Student's t-test. ns, not significant. 